\title{
Evaluation air void content of drilled porous asphalt mixture cores using non-destructive X-ray computed tomography
}

\author{
T. Vieira \\ Swedish National Road and Transport Research Institute, Linköping, Region Östergötland, Sweden
}

J. Lundberg

Division of Transport and Roads, Department of Technology and Society, Lund University, Lund, Sweden

O. Eriksson

Swedish National Road and Transport Research Institute, Linköping, Region Östergötland, Sweden

\author{
A. Guarin \& S. Gong \\ Division of Building Materials, Department of Civil and Architectural Engineering, KTH - Royal Institute \\ of Technology, Stockholm, Sweden \\ S. Erlingsson \\ Swedish National Road and Transport Research Institute, Linköping, Region Östergötland, Sweden \\ Division of Building Materials, Department of Civil and Architectural Engineering, KTH - Royal Institute \\ of Technology, Stockholm, Sweden \\ Faculty of Civil and Environmental Engineering, University of Iceland, Reykjavik, Iceland
}

\begin{abstract}
Tomography technology is not usual when investigating drilled asphalt cores properties. Currently, there is no internationally recognised standard tomography method for asphalt pavements. Tomography provides, however, a non-destructive alternative to traditional, usually destructive, testing of drilled cores. Furthermore, tomography offers possibilities, which traditional laboratory analyses do not. It is not straightforward to distinguish mastic and aggregates in tomography results while air void content is less difficult to assess. To have a more reliable assessment of drilled cores properties found by tomography, the method must be carefully planned, executed, and the results compared to those of traditional laboratory methods. In this work, analysis was carried out using six in-situ drilled cores from a double layered porous asphalt pavement. This allowed a comparison of tomography and a standardised conventional laboratory air void measurement on the exact same samples. Comparisons of the air voids found by tomography in all three directions were also carried out, estimating how anisotropic and heterogeneous the samples are, which is not possible using traditional laboratory tests. As few as four tomography slices can give enough precision in the determination of air void content for the porous layers. No more than eight slices per sample were needed in the suggested tomography method. The statistical results did not indicate that the air void content determined by tomography is different from the laboratory results.
\end{abstract}

Keywords: Tomography, non-destructive test, destructive test, air void content, porous asphalt drilled cores 


\section{INTRODUCTION}

Porous pavements were developed as a method to increase traffic safety by draining run-off water from the road surface and reduce splash and spray (Dawson et al., 2009). A more recent application of porous pavements has been to reduce tyre/road noise at its source, especially due to its high air void content (Sandberg and Ejsmont, 2002, Vieira et al., 2019, Vieira, 2020).Another potential application is to reduce non-exhaust particle emissions from the road surface/tyre interaction (Norman and Johansson, 2017, Lundberg et al., 2019, Lundberg, 2020). The noise reduction is relevant considering that excessive exposure to noise leads to health impacts, including increased blood pressure, sleep disturbance, among others (World Health Organization, 2011). Exposure to particles are also related to health impacts, including, for instance, respiratory diseases, cardiovascular diseases and cancer, among others (World Health Organization, 2013, World Health Organization, 2016).

Porous pavements, compared to traditional dense pavements, have certain requirements when it comes to the construction process. Under its lifecycle, the porous layers are subjected to clogging due to particles and road dust (Sandberg and Ejsmont, 2002, Norman and Johansson, 2017, Lundberg et al., 2020); this, in turn, decreases its acoustical benefits. Another important aspect is the decreased durability, especially where studded winter tyres are used (Sandberg and Ejsmont, 2002); this affects the maintenance requirements and activities, which also affects the resulting lifecycle costs and benefits.

In order to achieve the desired properties, it is important to have an appropriate quality control method. Presently, quality control is performed with traditional laboratory methods that are typically destructive and do not allow a detailed quantification of the internal structure, including the air void content. One relatively novel method is the use of X-ray computed tomography (CT-scan) to obtain a detailed three-dimensional description of the internal structure of drilled cores, however, there is currently no standard nor commonly accepted method to use tomography in order to evaluate air void content. It is also needed to explore how the air void content obtained by tomography compares to the results obtained by traditional laboratory methods.

To contribute to a better understanding of the tomography method, its applicability to evaluate air void content and validate it against traditional laboratory methods, six drilled cores were collected in Linköping, Sweden, from a double layered porous asphalt concrete (DLPAC). The investigation summarized here is intended to be a steppingstone to the development of a method to evaluate air void content using CT-scan, and, in continuation, other material properties, such as binder content, as well as degradation processes, e.g. aggregate cracking and clogging of air voids. This paper is based on the master thesis by Gong (2020) in which more details can be found.

The overall aim of this study is to develop a methodology to determine the air void content from drilled cores from porous asphalt pavements using CT-scanning; the results were validated by comparison to traditional laboratory methods. Furthermore, another specific objective was to determine different kinds of air void content, including total and interconnected air voids for each asphalt layer and drilled core.

\section{METHODS}

\subsection{Traditional laboratory determination of air void content and layer thickness}

The air void content is currently most commonly determined using traditional laboratory methods. In this study, the air void content was determined using the European standard EN 12697-8 (Swedish Standards Institute, 2019). In short, the air void content is determined by measuring the bulk and maximum density of the specimen. All traditional laboratory tests were carried out after the CT-scans, as destructive laboratory measurements were also performed, including binder determination. Layer thickness was 
measured using a measurement tape at four equidistant locations around the lateral surface of the drilled cores.

\section{$2.2 X$-ray computed tomography (CT-scanning)}

$\mathrm{X}$-ray CT scanning is a method which allows a three-dimensional determination of the internal structures of a specimen, in this case a drilled core of asphalt concrete (Guarin et al., 2012) in this case a drilled core of asphalt concrete. The X-ray CT-scanning device used in this study was the NSI X5000 located at KTH, laboratory of the Department of Civil and Architectural Engineering in Stockholm, Sweden; it is a seven-axis $\mathrm{X}$-ray imaging system with $225 \mathrm{kV}$ and $450 \mathrm{kV}$ X-ray tubes (focal spot sizes of $5 \mu \mathrm{m}$ and $400 \mu \mathrm{m}$, respectively). It has software for scan calibration, real time visualization and post-processing. A schematic view of the CT-scan components is displayed in Figure 1a. The CT-scan provides a series of grey-scale images which are stitched together to form a three-dimensional model of the specimen. This greyscale represents the density of the material where darker tones translates to lower densities. Figure $1 \mathrm{~b}$ shows the different cutting planes used.
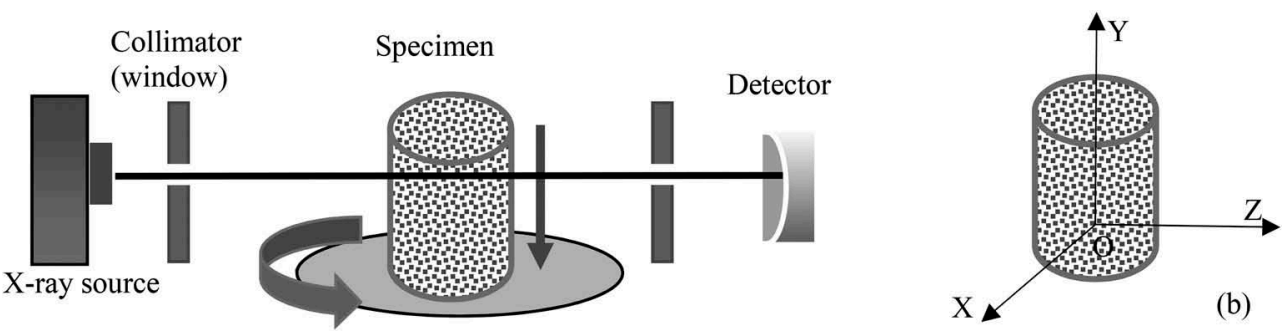

Figure 1. (a) Schematic of the X-ray CT-scan procedure. Figure based on Masad et al. (2002). (b) The different cutting planes defined by their normal direction, X, Y, and Z.

\subsection{CT-scan process and slice acquisition}

The general procedure includes sample preparation, calibration, data acquisition and reconstruction; further information can be found in Onifade (2013). In a few words, the specimen is scanned on a rotational table in the CT when geometrical configuration between x-ray source, sample, and detector panel (Figure 1b), as well as appropriate energy levels are verified. When the scan is completed, a series of greyscale $2 \mathrm{D}$ images is generated; the greyscale is related to density of the material, where darker tones mean lower densities. When the $2 \mathrm{D}$ images are put together, the three-dimensional object is generated

After scanning of the specimen, CT images are obtained using a reconstruction process which converts sinograms into two-dimensional slice images. With the used settings, each CTscan produced around 1500 slices in 16-bit scale per sample per direction.

\subsection{Image processing}

Image processing was required to differentiate between aggregates, mastic (asphalt binder and aggregate particles), and air voids, as well as, to determine the total and interconnected air void content. This was done using an open-source image processing software named ImageJ, v 1.0 from National Institutes of Health (Schneider et al., 2012). A flowchart depicting the process is presented in Figure 2 and it is described in more detail than here in Gong (2020). After the slice import, the image extension is changed to an 8-bit image format to allow 
further analysis. Cropping is applied to obtain the area of interest. The minimum auto threshold function is then applied to adjust the grey levels to more clearly identify the air voids in relation to the other material phases. A binary transformation was applied to the image thresholding results. Using this image, interconnected air voids were identified and classified according to the Lefebvre air void classification (Lefebvre, 1993). The interconnected air voids are, in this case, air voids connected to the surface as illustrated in Figure 3. The software measures the interconnected air voids area in each image and calculates the interconnected air voids content in the entire drill core.

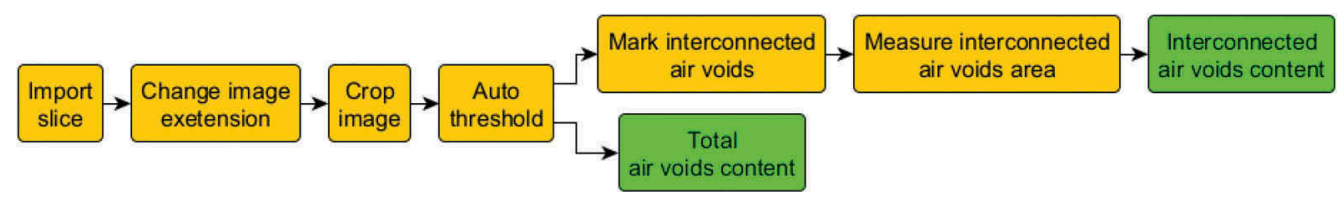

Figure 2. Flowchart describing the image analysis processing using the ImageJ software. Green colours mark output results.

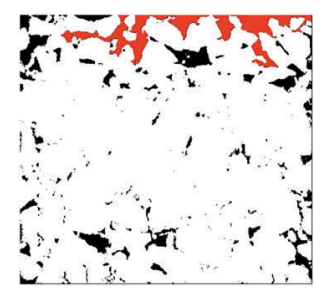

Figure 3. A binary transformation slice where the red areas are classified as interconnected air voids, black areas as non-interconnected air voids and white areas as mastic and aggregates. Figure source: Gong (2020).

\subsection{Qualitative analysis method}

Different slices can be selected in all three directions by adjusting the cutting plane. After reviewing all slices, representative slices for each drilled core were selected based on the following parameters: (i) interface between different layers (ii) air void content (relatively large or small), (iii) aggregate sizes, (iv) aggregate segregation, (v) aggregates' characteristics, e.g. cracking. Although criteria (iii), (iv), and (v) are not within the scope of this paper, they were used for the qualitative characterization of each drilled core.

\subsection{Quantitative analysis method}

\subsubsection{Layer thickness}

Layer thickness was measured both in the laboratory, and by using CT-scan slices. For the $\mathrm{CT}$-scan, slices in the X-direction was used. The layer thickness was measured on random points in the interface between layers in each CT-slice. This was repeated for five randomly chosen slices for each drilled core, and the average was calculated and used as the CT-scan layer thickness. 


\subsubsection{Air void content}

The quantitative analysis was done by investigating the effect of number of asphalt layers, number of slices from the CT-scan data, different analysis directions and combinations of them, on the calculated air voids content. Regression analysis was performed for each combination regarding air void content between CT-scan $\left(e_{C T}\right)$ and traditional laboratory methods $\left(e_{l a b}\right)$ where traditional laboratory was used as explanatory variable and CT-scan as response variable:

$$
E\left[e_{C T}\right]=\beta_{0}+\beta_{1} \cdot e_{l a b}
$$

where $E\left[e_{c t}\right.$ is the expected value of $e_{c t}$ for the given value of $e_{l a b}, \beta_{1}$ is the slope and $\beta_{0}$ is the intercept. For each combination three separate hypotheses $H$ were tested:

$$
\begin{gathered}
H_{1}: \beta_{0}=0 \\
H_{2}: \beta_{1}=1 \\
H_{3}: \beta_{0}=0 \text { and } \beta_{1}=1
\end{gathered}
$$

Hypothesis $H_{1}$ and $H_{2}$ were tested using two-tailed t-test. $H_{3}$ were tested using partial F-test. For all test, the significance level of $5 \%$ was used.

The Evaluation Index $(E I)$ was used to evaluate the accuracy using the slope and intercept of the regression analyses:

$$
E I=\left(\hat{\beta}_{1}-1\right)^{2}+\left(\hat{\beta}_{0}-0\right)^{2}+\left(R^{2}-1\right)^{2}
$$

where $\hat{\beta}_{1}$ is the estimated slope, $\hat{\beta}_{0}$ is the estimated intercept and $R^{2}$ is the coefficient of determination. However, this evaluation has the limitation of not being scale invariant.

\section{SITE AND DRILLED CORES DESCRIPTION}

\subsection{Site}

The investigated DLPAC was located at Industrigatan in northeast Linköping, Sweden (Figure 4). It was constructed in two layers during august 2018. The layers were designed with an intended air voids content of $23.1 \%$. The top layer and bottom layer were designed to have a thickness of $25 \mathrm{~mm}$ and $55 \mathrm{~mm}$ respectively and a maximum aggregate size of $11 \mathrm{~mm}$ and $16 \mathrm{~mm}$ respectively. The DLPAC was built on top of part of the old dense pavement at the site. The road section is about 600 meters long with an average annual daily traffic of about 14,700 vehicles of which about $7 \%$ are heavy vehicles. In Sweden, winter tyres (non-studded and studded) are mandatory during December through March given winter conditions, and studded tyres are common on light vehicles. The studded tyre share at Industrigatan was measured to between $51 \%$ and $66 \%$ for the light vehicles. 


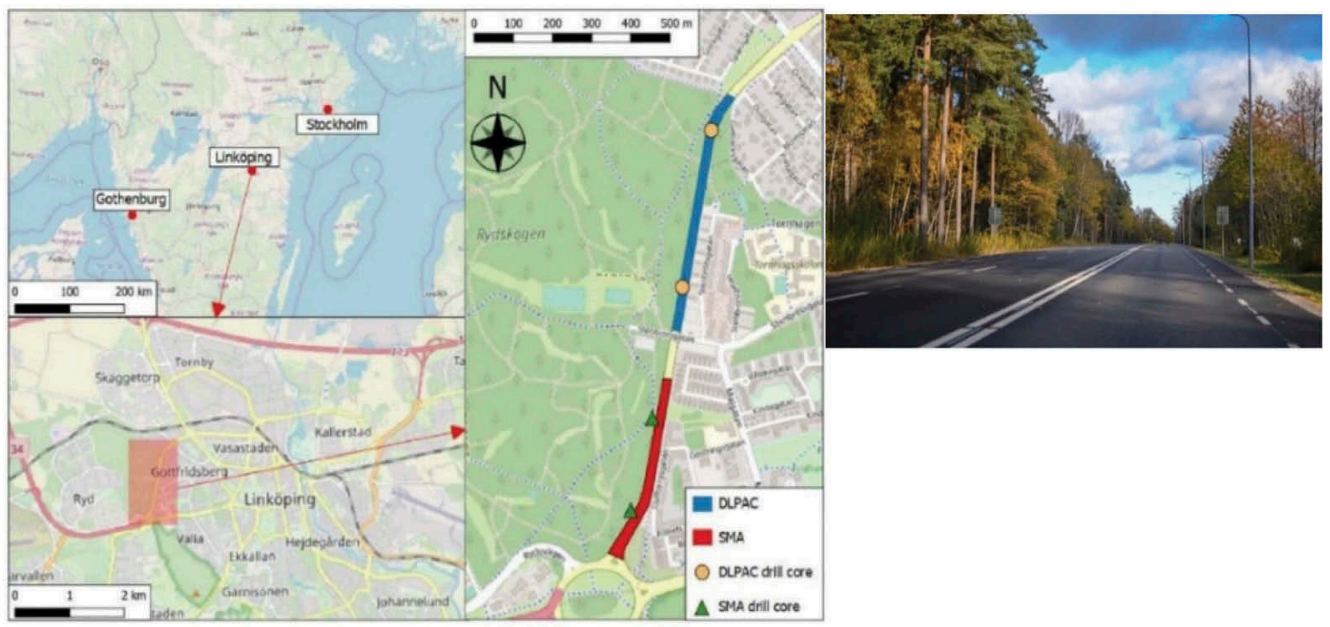

Figure 4. Left: Location of the double layered porous asphalt section in Linköping, Sweden. Adapted from Lundberg et al. (2020). Right: photo of Industrigatan in the northern driving direction at the double layered porous asphalt.

\subsection{Drilled cores}

Six drilled cores were taken for this study. Three drilled cores were taken each at the north and the south site of the DLPAC in the northbound direction (see Figure 4). At each site, one drilled core was taken per wheel track and one between wheel tracks. The drilled cores were taken in early November 2018, approximately two months after construction.

\section{RESULTS AND DISCUSSION}

\subsection{Traditional laboratory determination of air void content}

The results of the laboratory assessment of the drilled cores are presented in Table 1; it can be seen that the top layer is closer to the intended design with air void content between 18.6 $23.4 \%$ compared to the intended $23.1 \%$. The bottom layer, however, is far from the intended design with an air void content between $6.1-10.8 \%$ compared to the intended $23.1 \%$. This is visible from the CT-scans, as illustrated in Figure 5. Also, the layer thickness deviates from the intended design, with larger variations for the bottom layer. The binder content is instead close to the intended design.

Table 1. Comparison between the intended design values and the laboratory determination for air void content, layer thickness and binder content.

\begin{tabular}{|c|c|c|c|c|c|c|}
\hline \multirow[t]{2}{*}{ Layer } & \multicolumn{2}{|c|}{ Air void content $[\%]$} & \multicolumn{2}{|c|}{ Layer thickness [mm] } & \multicolumn{2}{|c|}{ Binder content $[\%]$} \\
\hline & Design value & $\begin{array}{l}\text { Drilled core } \\
\text { analysis }\end{array}$ & Design value & $\begin{array}{l}\text { Drilled core } \\
\text { analysis }\end{array}$ & Design value & $\begin{array}{l}\text { Drilled core } \\
\text { analysis }\end{array}$ \\
\hline Тор & 23.1 & $18.6-23.4$ & 25 & $20-30$ & 6.6 & $6.0-6.3$ \\
\hline Bottom & 23.1 & $6.1-10.8$ & 55 & $35-60$ & 6.4 & $6.4-6.8$ \\
\hline
\end{tabular}




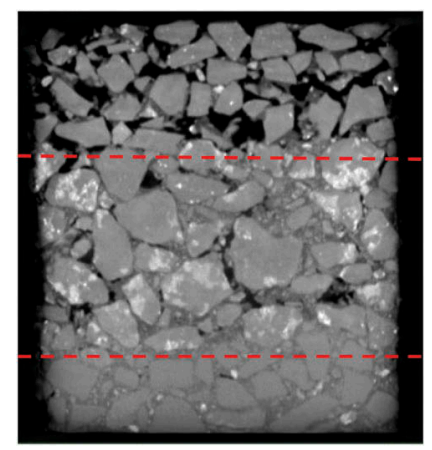

Figure 5. A CT-scan of one of the drilled cores. The top and middle layers are the DLPAC layers, while the bottom layer is the old dense pavement. The different grey-tones represents the material density where darker tones represent lower densities.

\subsection{Qualitative analysis}

The results from the qualitative analysis is summarised in Table 2. It was possible to detect different levels of segregation (for example Figure 6a) in the different drilled cores and the different layers within each core. It was also possible to visually estimate the different amounts of air void content within each core and layer (Figure 6a and b). Different interconnectivity levels were observed as well (Figure 6b). In general, both air void content and levels of interconnectivity are higher in the top layer compared to the bottom layer. Comparing the two sites, no clear difference is seen for the top layer regarding the air void content. However, at the northern site a more prominent densification in the bottom layer is visible. This is also true regarding the levels of interconnectivity. For segregation, a similar pattern is seen, although with less prominence. This indicates inhomogeneity of this test section, possibly due to the construction process or compaction by traffic.

Table 2. A qualitative comparison of similarities between the different drilled cores. $\mathrm{RW}=$ right wheel track, $\mathrm{BW}=$ between wheel track and $\mathrm{LW}=$ left wheel track.

\begin{tabular}{llllll}
\hline Drilled core & Air void content & \multicolumn{3}{l}{ Interconnectivity } & Segregation \\
\hline & Top & Bottom & Top & Bottom & Both layers \\
\cline { 2 - 6 } North RW & 4 & 4 & 4 & 5 & 7 \\
North BW & 5 & 3 & 5 & 2 & 3 \\
North LW & 7 & 4 & 7 & 4 & 4 \\
South RW & 6 & 6 & 7 & 7 & 5 \\
South BW & 4 & 5 & 5 & 7 & 4 \\
South LW & 5 & 4 & 4 & 4 & 3 \\
\hline
\end{tabular}

Note: Lower values indicate less observed features and higher values indicate more observed features. 


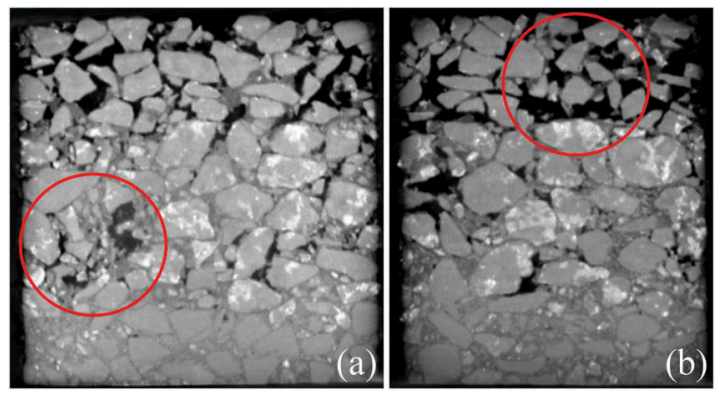

Figure 6. (a) Example on segregation and (b) interconnectivity. Figure source: Gong (2020).

\subsection{Quantitative analysis}

\subsubsection{Layer thickness}

The quantitative analysis allowed calculation of the layer thickness for all drilled cores. In average, the difference compared to the laboratory measurements was $8.9 \%$. However, the difference was found to have a large variation, between $0.1 \%$ and $30.6 \%$. This variation was due to the possibility to analyse the internal variation in layer thickness using the CT-scan method, which is not possible using traditional laboratory methods.

\subsubsection{Significance testing - difference between laboratory method and CT-scan}

Linear regression was performed on all combinations of layers and directions with number of slices varying between four, six and eight slices. An example of the results is presented in Figure 7, where R2, the intercept and the slope for the regression is presented. The statistical analysis of this regression analysis resulted in an intercept and a slope with p-values of 0.952 and 0.918 when testing hypotheses 1 and 2 respectively. Hypothesis 3 was tested with partial F-test but was not significant $(\mathrm{p}>0.999)$. The results, when considering different number of slices, different combination of layers and different combinations of directions are presented in Table 3 with the regression coefficients, i.e. the estimated slope, $\hat{\beta}_{1}$, the estimated intercept, $\hat{\beta}_{0}$, the coefficient of determination, $R^{2}$ and the evaluation index, $E I$. The t-tests and partial F-tests did not indicate that there is any difference between tomography and traditional laboratory results, and that four slices are enough to determine the air void content. However, the EI and $\mathrm{R}^{2}$ increase slightly when the number of slices is increased from 4 to 6 , and then to 8 .

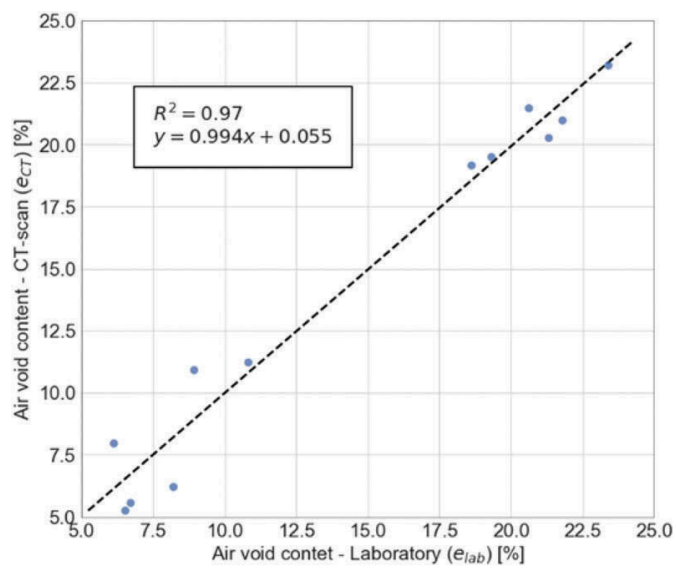

Figure 7. Air void content from the traditional laboratory method and from tomography (CT). Each point is the average air void content for the $\mathrm{X}, \mathrm{Y}$ and $\mathrm{Z}$ directions for six slices for each porous layer of the drilled cores. 
Table 3. Accuracy comparison of different combination. The optimal combination is highlighted by $\mathrm{a}$ *, being the $\mathrm{X}+\mathrm{Z}$ with 8 slices.

\begin{tabular}{lllcll}
\hline Directions & Number of slices & $\widehat{\beta}_{1}$ & $\widehat{\beta}_{0}$ & $\mathrm{R}^{2}$ & $\mathrm{EI}$ \\
\hline $\mathrm{X}$ & 4 & 0.9516 & 0.0022 & 0.9637 & 0.00366 \\
& 8 & 0.9628 & 0.0008 & 0.9667 & 0.00249 \\
$\mathrm{Y}$ & 4 & 1.0171 & -0.0048 & 0.9651 & 0.00153 \\
& 8 & 0.981 & 0.001 & 0.9732 & 0.00108 \\
$\mathrm{Z}$ & 4 & 0.9755 & 0.0016 & 0.9681 & 0.00162 \\
& 8 & 0.9949 & 0.0005 & 0.9681 & 0.00104 \\
$\mathrm{X}+\mathrm{Z}$ & 4 & 0.9636 & 0.0019 & 0.9681 & 0.00235 \\
& 8 & 0.9788 & 0.0007 & 0.9687 & 0.00143 \\
$\mathrm{Y}+\mathrm{Z}$ & 4 & 0.9963 & -0.0016 & 0.9684 & 0.00101 \\
& 8 & 0.9879 & 0.0008 & 0.9714 & 0.00097 \\
$\mathrm{X}+\mathrm{Y}$ & 4 & 0.9843 & -0.0013 & 0.9672 & 0.00132 \\
& 8 & 0.9719 & 0.0009 & 0.9705 & 0.00166 \\
$\mathrm{X}+\mathrm{Y}+\mathrm{Z}$ & 4 & 0.9814 & 0.0003 & 0.9687 & 0.00133 \\
& 8 & 0.9796 & 0.0008 & 0.9704 & 0.00129 \\
\hline
\end{tabular}

The air void content for each layer, as well as the interconnected air void content and the overall air void content are presented in Table 4 for each drilled core. These results are comparable to the ones obtained by traditional laboratory methods. This was previously shown in Figure 7.

Table 4. Results of average air void content [\%] using the directions indicated in parenthesis.

\begin{tabular}{lrrrrrr}
\hline Air void content & North RW & North BW & North LW & South RW & South BW & South LW \\
\hline Top layer (Average Y+Z) & 20.80 & 20.25 & 23.26 & 19.31 & 19.57 & 20.97 \\
Bottom layer (Average Y+Z) & 6.18 & 5.37 & 5.80 & 10.94 & 10.67 & 7.95 \\
Interconnected (Average X+Z) & 3.35 & 3.01 & 4.40 & 3.23 & 2.40 & 2.84 \\
Overall (Average X+Z) & 11.96 & 10.88 & 11.86 & 13.15 & 11.94 & 12.20 \\
\hline
\end{tabular}

\section{CONCLUSIONS AND RECOMMENDATIONS}

\subsection{Conclusions}

This study has developed a methodology for using CT-scanning for the determination of air void content of porous asphalt concrete drilled cores. Six drilled cores samples from Linköping, Sweden, were used for this purpose, in total 12 samples. From the results of this study, it was concluded that CT-scanning, in combination with image analysis were found to be suitable tools to analyse the air void content of drilled cores of porous pavements.

From the qualitative analysis it was noted that all drilled cores showed some common features: air void content in top layers were larger than bottom layers; interconnectivity between air voids were lower in the bottom layers than the top layers; and aggregate segregation was observed in both top and bottom layers. Sample characteristics that differed between samples were found to be: aggregate cracking was observed for some drilled cores in the bottommost layer; and in some samples air voids were observed in the interface between porous layers and porous and dense layers.

From the quantitative analysis it was shown that different kinds of air void content were found to be quantifiable using CT-scanning and the proposed methodology. Four slices were found to be sufficient to determine an accurate air void content compared to traditional laboratory results. 


\subsection{Recommendations and research needs}

Based on the study of drilled cores using CT scan, some recommendations and research needs are presented here. A method should be developed to determine not only air void content but also more asphalt mixture properties (e.g. aggregate size distributions, binder content, mastic content, aggregate content etc.), either individually or in combination with the air void structure. The relationship between different kinds of air void content and noise absorption, particle emissions should be evaluated, and a numerical relationship or model should be developed. The process of air void structure investigation using CT scanning should be developed to become automatized. The results presented here should be generalized by repeating the study on more drilled core samples, preferably using a mix of different types of porous pavements with different degrees of densification as well. The methodology developed in this study should also be applied to aggregate characterization or air void characterization of cement concrete pavements as well.

\section{ACKNOWLEDGEMENTS AND FUNDING}

The authors wish to acknowledge Leif Söderberg (NCC industry AB) who was hired to take the drilled cores samples. Also acknowledged are Ramudden AB which were hired to keep us safe during the acquisition of the drilled cores. Andreas Waldemarson (VTI) is acknowledged for performing the traditional laboratory measurements. This study was carried out as a master thesis project at KTH. The drilled cores acquisition was funded by Linköping.

\section{REFERENCES}

Dawson, A., Kringos, N., Scarpas, T. \& Pavsic, P. 2009. Chapter 5: Water in the Pavement Surfacing. In: Dawson, A. (ed.) Water in Road Structures - Movement, drainage effects. The Netherlands: Springer.

Gong, S. 2020. Investigation of Air Void Structure in Double Layer Porous asphalt based on X-ray Computed Tomography. Maste of Science, KTH Royal Institute of Technology: Stockholm, Sweden.

Lefebvre, G. 1993 Porous asphalt, Permanent International Association of Road, Congresses. Paris.

Lundberg, J. 2020. Road Surface and Tyre Interaction: Functional Properties affecting Road Dust Load Dynamics and Storage. KTH Royal Institute of Technology: Stockholm, Sweden.

Lundberg, J., Gustafsson, M., JanhÄll, S., Eriksson, O., Blomqvist, G. \& Erlingsson, S. 2020. Temporal variation of road dust load and its size distribution - a comparative study of a porous and a dense pavement Water, Air, \& Soil Pollution (submitted).

Lundberg, J., Vieira, T., Blomqvist, G., Gustafsson, M., JanhÄll, S., Genell, A. \& Erlingsson, S. 2019 PM10 Emissions from a Porous Pavement, and Implication for Noise Reduction, Initial Results. European Aerosol Conference, 2019 of Conference Gothenburg, Sweden.

Masad, E., Jandhyala, V. K., Dasgupta, N., Somadevan, N. \& Shashidhar, N. 2002. Characterization of Air Void Distribution in Asphalt Mixes using X-ray Computed Tomography. Journal of Materials in Civil Engineering, 14, pp. 122-129. doi: 10.1061/(ASCE)0899-1561(2002)14:2(122).

Norman, M. \& Johansson, C. 2017 Emission of PM10 and coarse particles from "silent" asphalt. 22nd International Transport and Air Pollution Conference 2017 of Conference Zürich, Switzerland.

Sandberg, U. \& Ejsmont, J. A. 2002. Tyre/road noise reference book, Kisa, Sweden, Informex.

Swedish Standards Institute 2019, SS-EN 12697-8: Bituminous mixtures - Test methods - Part 8: Determination of void characteristics of bituminous specimens. Stockholm, Sweden: Swedish Standards Institute.

Vieira, T. 2020. Tyrelroad interaction: A holistic approach to the functional requirements of road surfaces regarding noise and rolling resistance. 2013 Doctoral thesis, comprehensive summary, KTH Royal Institute of Technology: Stockholm.

Vieira, T., Lundberg, J., Genell, A., Sandberg, U., Blomqvist, G., Gustafsson, M., JanhÄll, S. \& Erlingsson, S. 2019 Porous pavement for reduced tyre/road noise and improved air quality - Initial results from a case study. In: Montreal, Icsv26 Local Committee In (ed.) International Congress on Sound and Vibration, 2019 of Conference Montreal, Canada. Canadian Acoustical Association, p. pp. 8. 
World Health Organization. 2011 Burden of disease from environmental noise: quantification of healthy life years lost in Europe. Copenhagen, Available at: http://www.euro.who.int/_data/assets/pdf_file/0008/ 136466/e94888.pdf.

World Health Organization. 2013 WHO 2013. Review of evidence on health aspects of air pollution REVIHAAP project: Final technical report. Copenhagen, Denmark.

World Health Organization. 2016 Ambient air pollution: A global assessment of exposure and burden of disease. Geneva, Switzerland. 